\title{
POUR UNE HISTOIRE CULTURELLE DE LA DIPLOMATIE. PRATIQUES ET NORMES DIPLOMATIQUES AU XIX ${ }^{E}$ SIĖCLE
}

Renaud Meltz, Isabelle Dasque

Armand Colin | « Histoire, économie \& société »

2014/2 33e année | pages 3 à 16

ISSN 0752-5702

ISBN 9782200929336

Article disponible en ligne à l'adresse :

https://www.cairn.info/revue-histoire-economie-et-societe-2014-2-page-3.htm

\section{Pour citer cet article :}

Renaud Meltz, Isabelle Dasque« Pour une histoire culturelle de la diplomatie. Pratiques et normes diplomatiques au $\mathrm{XIX}^{\mathrm{e}}$ siècle », Histoire, économie \& société 2014/2 (33e année), p. 3-16.

DOI 10.3917/hes.142.0003

Distribution électronique Cairn.info pour Armand Colin.

(c) Armand Colin. Tous droits réservés pour tous pays.

La reproduction ou représentation de cet article, notamment par photocopie, n'est autorisée que dans les limites des conditions générales d'utilisation du site ou, le cas échéant, des conditions générales de la licence souscrite par votre établissement. Toute autre reproduction ou représentation, en tout ou partie, sous quelque forme et de quelque manière que ce soit, est interdite sauf accord préalable et écrit de l'éditeur, en dehors des cas prévus par la législation en vigueur en France. Il est précisé que son stockage dans une base de données est également interdit. 


\title{
Pour une histoire culturelle de la diplomatie. Pratiques et normes diplomatiques au XIX ${ }^{\mathrm{e}}$ siècle
}

\author{
par Renaud Meltz et Isabelle Dasque
}

\section{Introduction}

L'histoire de la diplomatie au XIX ${ }^{\mathrm{e}}$ siècle n'aurait-elle pas droit au bénéfice du renouvellement historiographique et épistémologique que connaît l'histoire des relations internationales, à l'instar des autres champs de la discipline historique ${ }^{1}$ ?

L'histoire diplomatique du XIX ${ }^{\mathrm{e}}$ siècle mérite pourtant d'être revisitée, réinvestie, vivifiée par de nouveaux regards. Depuis plusieurs décennies, le renouvellement de l'histoire des relations internationales met en œuvre le programme ambitieux qu'avaient tracé les figures tutélaires de Pierre Renouvin et Jean-Baptiste Duroselle dans les années $1960^{2}$. Dès les années 1950, le premier (1893-1974) avait déduit de sa confrontation avec l'histoire immédiate, et l'entêtant débat sur l'origine de la Grande Guerre, la nécessité de prendre en compte les transformations de l'économie et autres « forces profondes ». La vaste fresque dont il était maître d'œuvre, déroulait sur plusieurs siècles une Histoire des relations internationales sensible à cette préoccupation. Le cadet, né une génération plus tard (19171994), ouvrait l'histoire des relations internationales aux progrès des sciences sociales et humaines, pour une lecture plus complexe de la psychologie de l'homme d'État et des processus de décision. Aussi bien, l'État demeurait au centre de cette nouvelle approche des relations internationales, pluridisciplinaire et pluricausale.

En formulant la proposition précoce et novatrice d'une histoire politique ouverte aux perspectives économiques, sociales et culturelles, Renouvin et Duroselle ont fait souffler un vent nouveau sur l'histoire dite diplomatique, telle que la pratiquait en France un

1. Pour un bilan historiographique, Lucien Bély et Georges-Henri Soutou, « Les relations internationales », dans Les historiens français à l'œeuvre, 1995-2010, dir. Jean-François Sirinelli, Pascal Cauchy, Claude Gauvard, PUF, 2010, p. 261-286 ; Robert Frank (dir.), Pour l'histoire des relations internationales, PUF, 2012, p. 5-40 ; id., «Penser historiquement les relations internationales », Annuaire français des relations internationales, 2003, vol. IV, p. 42-65 ; Stanislas Jeannesson, « Diplomatie et politique étrangère de la France contemporaine : un bilan historiographique depuis 1990 », dans Nouvelles Approches en histoire de la France contemporaine, numéro spécial d'Histoire, Économie et Société, 2012/2, p. 87-98.

2. Pierre Renouvin et Jean-Baptiste Duroselle, Introduction à l'histoire des relations internationales, Armand Colin, 1964. 
Albert Sorel ${ }^{3}$. Ils renouvelaient cette histoire fidèle aux règles de l'École méthodique, limitée au récit d'événements fabriqués par les seuls acteurs des négociations politiques, ministres et diplomates ${ }^{4}$. Depuis trois générations que s'est trouvée fondée ce qu'il est convenu d'appeler l'École française de l'histoire des relations internationales, sans que ses plus récents rameaux en soient des disciples toujours revendiqués, formant un bouquet monochrome, force est de constater que l'histoire diplomatique n'en a pas suffisamment tiré les fruits. C'est particulièrement vrai pour le $\mathrm{XIX}^{\mathrm{e}}$ siècle, qui a pourtant suscité des travaux novateurs dans le domaine domestique, en bénéficiant des questionnements et des outils de l'histoire culturelle 5 .

On connaît les reproches sarcastiques et heuristiques formulés par Lucien Febvre dans l'entre-deux-guerres, contre l'histoire diplomatique alors pratiquée, myope dans sa lecture des mouvements de l'histoire, incapable de faire droit au renouvellement de son objet : la diplomatie de la place publique, comme on disait dans les années 1920, remplaçait celles des chancelleries, « des Cours et des Cabinets », tandis que la pression de l'économie diminuait l'emprise du politique sur le fait diplomatique. Tout cela, s'indignait Lucien Fevbre, sans modifier les habitudes des historiens de la diplomatie ! La prise en considération, par Renouvin et Duroselle, des « forces profondes » à l'œuvre dans les relations internationales, a répondu partiellement à l'exigence formulée par l'École des Annales pour l'histoire des relations internationales - sans donner satisfaction au souhait de renouvellement des sources, que Lucien Febvre ne voulait pas limiter aux documents diplomatiques officiels ${ }^{6}$.

Cet élargissement, réclamé en faveur d'une histoire renouvelée des rapports entre les États, a porté ses premiers fruits dès les années 1970. Sur le plan théorique, Jean-Baptiste Duroselle a couronné un premier cycle de ce renouvellement, en 1981, avec Tout empire périra : théorie des relations internationales. En termes de travaux pratiques, les facteurs d'ordre matériels, géographiques ou économiques ont été pris en considération par les thèses magistrales de Jacques Thobie, Pierre Guillen, Raymond Poidevin, René Girault ou Pierre Milza. Dans le domaine des dimensions immatérielles, celui des « tempéraments nationaux » et des « mentalités collectives », les générations suivantes, aussi entremêlées qu'à l'époque de Renouvin et Duroselle, ont adjoint le poids des représentations et des perceptions - on songe naturellement aux travaux de René Girault et Robert Frank ${ }^{7}$. Plus

3. « [...] de par leurs objets, l'histoire des relations économiques et de la finance internationales, l'histoire des migrations, ces « forces profondes » où le national et l'international sont indissociables, ont devancé le tournant transnational des années 1990. »Sabine Dullin, Pierre Singaravelou, « le débat public : un objet transnational ? », Revue Monde(s), Histoire Espaces Relations, 2012/1, n 1, p. 13.

4. Gérard Noiriel, Qu'est-ce que l'histoire contemporaine, Hachette, 1998, p. 51-52.

5. Nous pensons par exemple, sans souci d'exhaustivité à Pierre Karila-Cohen, L'État des esprits. L'invention de l'enquête politique en France (1814-1848), Rennes, Presses universitaires de Rennes (PUR), 2008 ; Emmanuel Fureix, La France des larmes. Deuils politiques à l'âge romantique (1814-1840), Champ Vallon, 2009 ; Vincent Robert, Le temps des banquets. Politique et symbolique d'une génération (1818-1848), Publications de la Sorbonne, 2009 ; Corinne Legoy, L'enthousiasme désenchanté. Éloge du pouvoir sous la Restauration, Paris, Société des études robespierristes, 2010 ; Judith Lyon-Caen, La Lecture et la Vie. Les usages du roman au temps de Balzac, Tallandier, 2006 ; Matthieu Brejon de Lavergnée, La société de Saint-Vincent de Paul au $X I X^{e}$ siècle. Un fleuron du catholicisme social, Éd. du Cerf, 2008 ; François Jarrige, Au temps des « tueuses de bras ». Les bris de machines à l'aube de l'ère industrielle (1780-1860), Rennes, PUR, coll. « Carnot », 2009.

6. Lucien Febvre, Combats pour l'histoire, « contre l'histoire diplomatique en soi », Armand Colin, 1953. François Dosse, Christian Delacroix, Patrick Garcia, Les courants historiques en France, XIX ${ }^{e}-X X^{e}$ siècles, «Folio Histoire », 2007, p. 201-207.

7. René Girault, Conscience et identité européenne au XX $X^{e}$ siècle, Hachette, $1994 ;$ id., Être historien des relations Internationales, Publications de la Sorbonne, 1998 ; Robert Frank (dir.), Images et imaginaire dans les 
récemment, l'ouverture disciplinaire a produit ses effets au bénéfice d'une approche des relations internationales et de ses acteurs tenant de l'histoire sociale ${ }^{8}$, voire de l'anthropologie ${ }^{9}$.

Ce renouveau a posé les jalons d'une histoire sociale et culturelle de la diplomatie ${ }^{10}$. Cette dernière est appelée à contribuer à l'ambition actuelle d'une histoire connectée et croisée. Si l'on définit la diplomatie par trois fonctions essentielles de mise en relation (que ce soit entre les États, les peuples ou les sociétés), la négociation, la représentation et l'information, il est de bonne logique d'attendre de l'histoire diplomatique une contribution décisive à l'effort de connexion des historiographies nationales. Stimulés par l'élargissement de leur champ, il serait fâcheux que les historiens des relations internationales en viennent à oublier le potentiel épistémologique de l'histoire diplomatique. La belle ensommeillée ne demeure-t-elle pas la mieux placée pour répondre à l'exigence, aussi légitime que rabâchée, à l'heure de la troisième mondialisation, d'une histoire globale, faute d'historiographies unifiées ou d'épistémologies partagées ${ }^{11}$ ?

Nous proposons, à l'occasion de ce numéro spécial d'HES, de contribuer avec modestie, mais avec ardeur, à l'impulsion que nous estimons nécessaire pour un double renouvellement de l'histoire diplomatique du XIX ${ }^{\mathrm{e}}$ siècle. Renouvellement historiographique, d'abord. L'histoire du XIX ${ }^{\mathrm{e}}$ siècle a été largement revisitée, depuis une décennie, par l'histoire culturelle du politique, grâce à des approches neuves, touchant généralement le champ national, sans que des objets invisibles à force d'être familiers, comme l'État dans les relations internationales, en aient bénéficié ${ }^{12}$. Renouvellement épistémologique, pour ce faire, en faveur d'une histoire diplomatique restée dans l'ombre des questionnements féconds de l'histoire des relations internationales.

Nous avons choisi d'alimenter cette double ambition en soumettant l'histoire diplomatique du XIX ${ }^{\mathrm{e}}$ siècle à la question d'une notion aussi antique que stimulante : celle de la

relations internationales depuis 1938, Cahiers de 1'IHTP, n 28, juin 1994 ; Maria M. Benzoni, Robert Frank, Silvia M. Pizzetti (dir.), Image des peuples et histoire des relations internationales du XVIII e siècle à nos jours, Milan-Paris, UNICOPLI-Publications de la Sorbonne, 2008.

8. Claire Bénazet, Ambassadeurs et ministres de France de 1748 à 1791. Étude institutionnelle et sociale, Thèse de l'École des Chartes, 1982 ; Anne Mézin, Les consuls de France au siècle des Lumières (1715-1792), Ministère des Affaires étrangères, Direction des archives et de la documentation, 1997 ; Didier Ozanam, Les diplomates espagnols du XVIII siècle : introduction et répertoire biographique : 1700-1808, Madrid, Casa de Velázquez, 1999 ; Sabine Dullin, Des hommes d'influences : les ambassadeurs de Staline en Europe, 1930-1939, Payot, 2001 ; Isabelle Dasque, A la recherche de Monsieur de Norpois : les diplomates de la République (18711914), Thèse de l'Université Paris-Sorbonne, 2005 ; Peter Jackson, « Tradition and adaptation : the social universe of the French Foreign ministry in the era of the First world war », French History, 2010/2, vol. 24.

9. Laurence Badel, «Conclusion. Le verbe et le corps : anthropologie du diplomate écrivain », dans Écrivains et diplomates. L'invention d'une tradition $X I X^{e}-X X^{e}$ siècles, Dir. Laurence Badel, Gilles Ferragu, Stanislas Jeannesson et Renaud Meltz, Armand Colin, 2012, p. 398-408.

10. Lucien Bély, Espions et ambassadeurs au temps de Louis XIV, Fayard, 1990 ; Claire Gantet, La paix de Westphalie (1648). Une histoire sociale, XVII -XVIII siècles, Belin, 2001 ; Isabelle Dasque, A la recherche de Monsieur de Norpois, op. cit. ; Georges-Henri Soutou et Jean-François Sirinelli, Culture et guerre froide, PUPS, 2008 ; Anne Dulphy, Robert Frank, Marie-Anne Matard-Bonucci, Pascal Ory (dir), Les relations culturelles internationales au XX siècle. De la diplomatie culturelle à l'acculturation, Bruxelles, PIE Peter Lang, 2010. Laurence Badel, Gilles Ferragu, Stanislas Jeannesson et Renaud Meltz (dir.), Écrivains et diplomates, op. cit.

11. Patrick Boucheron rappelle que Global History et Connected Histories « ne partagent rien ou presque du point de vue méthodologique ou épistémologique », Patrick Boucheron, « L'entretien du monde », Pour une histoire monde, PUF, 2013, p. 6.

12. Aux ouvrages cités plus haut, ajoutons l'article-manifeste d'Eric Anceau, « Pour une histoire politique totale de la France contemporaine », dans Nouvelles approches en histoire de la France contemporaine, numéro spécial d'Histoire, Économie et Société, 2012/2, p. 116-117. 
morale. La notion, pour paraitre désuète, ou décalée de ces enjeux, relevant a priori de l'histoire des idées, de l'histoire religieuse ou de l'histoire culturelle, a nourri un projet de séminaire, puis suscité l'organisation d'un colloque, tenu en Sorbonne en 2012. Cette rencontre d'historiens venus d'horizons divers, autour du thème imposé, « morale et diplomatie au XIX ${ }^{\mathrm{e}}$ siècle », a validé la pertinence de ce rapprochement. Les articles réunis dans ce numéro sont plus ou moins directement issus de ce colloque. Ils reprennent à leur compte les questionnements que propose la notion de morale, entendue comme raison pratique autant que corpus de codes et de valeurs.

Aussi bien, la morale permet-elle de traverser les ambitions que nous nourrissons pour l'histoire diplomatique du XIX ${ }^{\mathrm{e}}$ siècle.

Sur le plan historiographique, on voit bien la fécondité de la notion : le XIX $\mathrm{X}^{\mathrm{e}}$ siècle ne cesse de ressasser les grands bouleversements cristallisés par la révolution française. Ce bouleversement pose la question des valeurs défendues par les États mais aussi par les diplomates qui les représentent, sans compter les nouveaux acteurs diplomatiques qui portent leurs propres exigences morales. Au titre de l'histoire globale, qui est l'horizon historiographique actuel, la notion de morale n'est pas moins heuristique : nous pensons aux confrontations et aux circulations des valeurs et des normes qui se jouent avec la mise en relation accélérée des différentes aires de civilisation, au siècle des impérialismes et des Empires. Le renouvellement conceptuel que nous appelons de nos vœux s'articule autour de la question des pratiques, grâce aux outils de l'histoire sociale et de l'anthropologie ${ }^{13}$. On voit bien en quoi cette question se trouve liée à la notion de morale, entendue comme raison pratique, depuis l'ethos du diplomate à la bonne conduite des États. C'est naturellement ce renouvellement épistémologique, nourri par la confrontation disciplinaire, qui peut justifier, in fine, l'intention revendiquée au seuil de ce dossier, en faveur d'une nouvelle historiographique de l'histoire diplomatique.

Pourquoi le renouvellement de la recherche en relations internationales ne profiterait-il pas à l'histoire diplomatique du XIX ${ }^{\mathrm{e}}$ siècle, demandions-nous ? La question de la morale offre une occasion de la revitaliser en l'ouvrant à d'autres champs disciplinaires comme l'histoire des idées, la philosophie morale ou les sciences politiques.

\section{Un renouvellement historiographique au prisme de la morale : les acteurs de la diplomatie au xix ${ }^{\mathrm{e}}$ siècle, normes et valeurs}

Abandonné aux historiens classiques de l'époque du positivisme, pris en tenaille entre le foisonnement des études sur la diplomatie d'Ancien Régime et celles sur le $\mathrm{XX}^{\mathrm{e}}$ siècle, le $\mathrm{XIX}^{\mathrm{e}}$ siècle, disions-nous, est resté pendant longtemps en marge du dynamisme touchant l'histoire des relations internationales. L'histoire de la diplomatie française suscite par exemple peu de vocations ; ses progrès ne bénéficient pas d'une bonne visibilité académique, que l'on entende par-là audience scientifique, présence éditoriale, ou transmission

13. Voir, du côté des politistes, Christer Jönsson et Martin Hall, Essence of Diplomacy, Houdsmille, Macmillan, 2005 ; Paul Sharp et Geoffrey Wiesmann, The Diplomatic Corps as an Institution of International Society, Houdsmille, Macmillan, 2007 ; Guillaume Devin, Sociologie des relations internationales, La Découverte, 2001 ; Marie-Christine Kessler, Les ambassadeurs, Les Presses de Sciences-Po, 2012. Chez les sociologues, Françoise Pitotet, Marc Loriol, David Defolie, Splendeurs et misères du travail des diplomates, Paris, Hermann, 2013 ; Meredith Kingston de Leusse, Diplomate. Une sociologie des ambassadeurs, L'Harmattan, 1997 ; Marc Loriol, «Les enjeux actuels de l'évolution des métiers de la diplomatie », Les cahiers Irice 2009/1 (n 3), p. 81-102 ; Du côté des historiens inspirés par une démarche culturaliste, voir par exemple Markus Mösselang et Torsten Riotte, The Diplomats's World. A cultural History of Diplomacy, 1815-1914, Oxford, Oxford University Press, 2008. 
pédagogique $^{14}$. Pourtant, cette histoire a bénéficié d'un premier rafraîchissement historiographique, venu d'outre-Atlantique, grâce à l'approche systémique qui l'affranchit de la seule étude des rapports inter-étatiques. Les travaux de Paul Schroeder ${ }^{15}$, popularisés en France par Georges-Henri Soutou ${ }^{16}$, ont introduit une réflexion sur le fondement civilisationnel et idéologique, bref, sur le substrat organique que règle la mécanique nouvelle du multilatéralisme, à quoi l'on a trop réduit l'ordre européen né au Congrès de Vienne. Naturellement, au XIX ${ }^{\mathrm{e}}$ siècle, cet ordre organique n'a pas l'homogénéité ni la constance qu'il avait dans l'Europe de l'Ancien Régime, où les valeurs chrétiennes primaient sur toutes les autres, en dépit de « l'étonnante mais indéniable reviviscence des philosophies antiques » (cf. article de S. De Franceschi).

Le $\mathrm{XIX}^{\mathrm{e}}$ siècle est traversé par un mouvement de balancier entre deux revendications à régénérer l'ordre international, au nom de morales distinctes. Le Congrès de Vienne, prolongé par la Sainte-Alliance, renoue avec la foi en l'ordre divin pour pacifier et stabiliser le vieux continent. L'héritage des Lumières et de la Révolution alimente la contestation de l'ordre de Vienne. Aux frontières de 1815, aux relations inter-étatiques, peu soucieuses des aspirations populaires, libéraux, démocrates et républicains opposent les principes nouveaux, dont la souveraineté nationale est la pierre de touche. Ces deux conceptions, l'une inspirée du décalogue, l'autre de l'humanisme des Lumières, parcourent tout le XIX ${ }^{\mathrm{e}}$ siècle ; elles trouvent une application dans tous les champs de la vie politique, sociale, culturelle et leur inscription dans le droit des États. Mais il serait par trop paresseux d'opposer seulement ces morales, et de leur distribuer le siècle, à l'une la restauration de l'Ancien Régime, à l'autre l'avènement de la Démocratie, pour imputer subrepticement la dissolution de l'ordre européen à l'affaiblissement de la première (une certaine historiographie, hostile à la République, donne parfois ce sentiment).

Certes, la diplomatie du premier XIX ${ }^{\mathrm{e}}$ siècle fonde le concert européen, en puisant avant tout (sans ignorer les Lumières !) aux principes de légitimité, de respect des droits des souverains et du droit des gens traditionnel. Pour autant, la Sainte-Alliance ne rechigne pas à la guerre, afin de maintenir l'ordre de Vienne, ce dont témoigne, parmi bien d'autres expéditions militaires, l'intervention française en Espagne (cf. article E. Larroche). C'est le libéralisme des monarchies constitutionnelles, celui de Castlereagh, puis de Guizot, qui tempère les velléités interventionnistes de Metternich.

Certes, le principe de souveraineté nationale, qui progresse à mesure que se répand le modèle de l'État-nation, mine le concert européen inspiré par les principes chers à Metternich. Peut-on pour autant imputer la constitution de blocs antagonistes, dont

14. S. Jeannesson, « Diplomatie et politique étrangère de la France contemporaine », art. cit., p. 97. Parmi les travaux portant sur la diplomatie française au XIX ${ }^{\mathrm{e}}$ siècle, Jacques-Alain de Sédouy, Chateaubriand : un diplomate insolite, Perrin, 1992 ; id., Le Congrès de Vienne : l'Europe contre la France : 1812-1815, Perrin, 2003 ; id., Le concert européen : aux origines de l'Europe, 1814-1914, Fayard, 2009 ; Raymond Bourgerie, Magenta et Solferino (1859) : Napoléon III et le rêve italien, Paris Economica, 1993 ; Emmanuel de Waresquiel, Talleyrand: le prince immobile, Fayard, 2003 ; Nicolas Jolicoeur, La politique française envers les États pontificaux sous la monarchie de Juillet et la Seconde république, 1830-1851, Bruxelles, PIE-Peter Lang, 2008 ; Servane Marzin, L'Europe de François Guizot (1840-1848), thèse de l'Université de Paris Nanterre la Défense, 2006 ; Yves Bruley, Le Quai d'Orsay impérial, Pedone, 2012 ; Emmanuel Larroche, L'expédition d'Espagne. 1823 : de la guerre selon la Charte, Rennes, PUR, 2013 ; Olivier Varlan, Armand-Louis de Caulaincourt, duc de Vicence (1773-1827) : étude d'une carrière diplomatique sous le Premier Empire, de la cour de Napoléon au ministère des Relations extérieures, Thèse de l'Université Paris-Sorbonne, 2013.

15. Paul Schroeder, The Transformation of European Politics 1763-1848, Oxford, 1994 ; id., « The nineteenth-Century International System : Changes in the structure », World Politics, 39, 1986-1987; id., « Did the Vienna system rest on a balance of Power ? », American Historical Review, 97, 1992, p. 683-706.

16. Georges-Henri Soutou, L'Europe de 1815 à nos jours, PUF, Nouvelle Clio, 2007. 
Bismarck fut le premier acteur, au principe de souveraineté nationale, alors que le chancelier de fer s'en proclamait le premier adversaire ? La tradition libérale n'a-t-elle pas son propre idéal d'ordre et de paix universels ? Ses plans de paix perpétuelle ${ }^{17}$, qui espèrent la paix mondiale grâce à la généralisation des régimes républicains, associant le peuple à la délibération politique ? Celui de Kant, publié en 1795, en constitue la matrice, ou du moins le modèle popularisé par les philosophes et les historiens des idées ${ }^{18}$. Les progrès du pacifisme internationaliste, plus ou moins synchrones à ceux des socialistes, dans chacun des États aux régimes représentatifs, ne puisent-ils pas à ces deux traditions chrétienne et libérale, trop schématiquement opposées ?

Une histoire diplomatique sensible à l'histoire des idées et à la philosophie politique, une histoire attentive au long terme, donnent à voir combien ces principes s'emmêlent comme ils s'opposent. Guillemette Crouzet montre dans ce dossier comment la GrandeBretagne, garante de l'ordre de Vienne, fonde son nouveau rapport au monde, après la perte des colonies d'Amérique, sur des valeurs issues du libéralisme des Lumières et diffusées par le courant abolitionniste anglais à partir de 1780/1800. Mais droits naturels et valeurs chrétiennes s'imbriquent pour inspirer ceux qui combattent la traite. Libéralisme, philanthropisme laïc et missionnaire se combinent, notamment dans le protestantisme anglais qui lutte contre la traite dans le Golfe Persique.

Bien entendu, les combats en faveur de la liberté de conscience et de commerce, pour le droit des peuples à disposer d'eux-mêmes ou contre la traite, s'inspirent, en Europe occidentale, des Lumières écossaises et continentales, bref, des valeurs que s'est choisie la Révolution française. La constitution d'un espace public européen permet l'émergence d'une première opinion publique internationale, traversée par les idéaux des Lumières, et les opinions qui contestent l'ordre de Vienne sont plus sonores que celles qui les défendent. Mais, aux côtés de la notion de droit des peuples à disposer d'eux-mêmes, qui est au cœur du combat philhellène ou pro-belge et polonais, la solidarité chrétienne joue un rôle considérable, parfois cumulatif, parfois dissocié. L'aspiration à l'émancipation de l'ensemble des peuples est associée à la revendication démocratique pour chacun des peuples ; mais les valeurs chrétiennes n'y sont pas étrangères comme le montre le moment 1848. Droits naturels et valeurs chrétiennes s'entremêlent dans des proportions variables, chez les républicains et les pacifistes.

Mieux : par un étrange paradoxe, cet État libéral, qui continue le grand processus de sécularisation de la politique initié par l'Humanisme et la réforme, pour dessiner les choses à très grands traits, renoue également avec la forme de prudence et le refus d'assumer une mission morale pratiquée par la Monarchie absolue. Ce qui revient à identifier d'étonnantes continuités de l'histoire politique, après l'événement singulier de la Révolution française, et son rejet de l'arbitraire d'un État monarchique, auquel on reprochait d'avoir expulsé de la sphère publique le jugement moral, propre à l'éthique chrétienne ${ }^{19}$. En somme, l'État libéral prolonge un rapport de déception avec le public, en refusant d'incarner des valeurs, fussent-elles libérales, dans sa politique étrangère, au profit de la défense d'intérêts, proportionnée aux moyens de la nation. Bien entendu, la délibération parlementaire, même dans un régime électoral faiblement représentatif, fait entendre la voix du peuple et marque

17. Marc Belissa, «Les projets de paix perpétuelle de l'abbé de Saint-Pierre à Jeremy Bentham », dans La cara oculta de la razon : locura, creencia y utopia, Cinta Canterla Gonzàlez (dir.), Universidad de Cadiz, Servicios de publicaciones, 2001, p. 61-74.

18. Marc Belissa et Florence Gauthier, «Kant, le droit cosmopolitique et la société civile des nations », Annales historiques de la Révolution française, 1999/3, p. 495-511.

19. Roger Chartier, Les origines culturelles de la Révolution française, Seuil, 2000, p. 294. 
les progrès de la rationalité collective contre la raison d'État. Reste que la revendication morale, portée par cet acteur insaisissable qu'est l'opinion publique, selon des valeurs difficiles à unifier selon les intérêts, les idéologies, les sentiments religieux, constate la déshérence d'un État moral et conteste sa politique étrangère, réduite à la défense de ses seuls intérêts nationaux (cf. article R. Meltz). Les dimensions morales, immatérielles, que sont l'honneur, la dignité ou la solidarité entre les peuples, sont prises en compte par ces nouveaux acteurs qui justifient que l'on parle de diplomatie des peuples.

À cet égard, deux champs se révèlent particulièrement féconds. D'une part, l'étude des mouvements de volontariat international armé, initiée par Ferdinand Boyer et relancée par Gilles Pécout ${ }^{20}$, qui s'inscrit dans une histoire de l'amitié politique entre les peuples ; d'autre part, sans que l'on puisse séparer absolument ces deux domaines, la question des opinions publiques, au siècle de la presse, qui offre un premier système médiatique où s'incarne une prétention à dire, à faire, bref à constituer une institution sociale de l'opinion publique.

Le premier est illustré par de nombreux travaux, qui portent sur les officiers de la Grande Armée $^{21}$, le mouvement philhellène ${ }^{22}$, le volontariat étranger pro-risorgimental ${ }^{23}$ ou l'épopée des volontaires garibaldiens, au secours des peuples en lutte pour leur indépendance $^{24}$. À côté du mouvement révolutionnaire ${ }^{25}$, s'impose une mobilisation internationale en faveur du légitimisme, celui des Bourbons de Naples contre le Risorgimento ${ }^{26}$, des Bourbons de France et d'Espagne ${ }^{27}$ ou encore au nom de la défense du pouvoir temporel du Pape ${ }^{28}$. D'autre part, la prise en compte des questions internationales par les opinions

20. Gilles Pécout, « The international armed volunteers : pilgrims of a transnational Risorgimento », dans International Volunteers and the Risorgimento, numéro spécial du Journal of Modern Italian Studies, vol. 14, issue 4, 2009, p. 413-426.

21. Walter Bruyère-Ostells, La Grande armée de la liberté, Taillandier, 2009.

22. Hervé Mazurel, Vertiges de la guerre. Byron, les philhellènes et le mirage grec, Les Belles Lettres, 2013. Antonis Liakos, L'Unificazione italiana e la grande idea, Florence, Alethaia, 1995 ; G. Pécout, « Philhellenism as a political freindship : Italian volunteers in $\mathrm{XIX}^{\mathrm{e}}$ th century Mediterranean », Journal of Modern Italian Studies, Vol. 9, issue 4, 2004, p. 405-427 ; id., « une amitié politique méditerranéenne : le philhellénisme italien et français au XIX ${ }^{\mathrm{e}}$ siècle », dans La democrazia radicale nell'Ottocento europeo. Annali della Fondazione Giangiacomo Feltrinelli-2003, dir. Maurizio Ridolfi, Milan, Feltrinelli, 2005, p. 81-106.

23. Anne-Claire Ignace, Des quarante-huitards français en Italie : étude sur la mobilisation de volontaires français pour le Risorgimento 1848-1849. Thèse de l'Université Panthéon-Sorbonne en cotulle avec l'Université de Pise, 2010 ; Ferdinand-Nicolas Göhde, « German volunteers in the armed conflicts of the Italian Risorgimento, 1834-1870 », dans International Volunteers and the Risorgimento, op. cit., p. 460-475.

24. G. Pécout, « Garibaldi e i garibaldini verso Creta nel 1866-1869 », Rassegna Storica del Risorgimento, 80, 1993, III, p. 316-343 ; Daria Ermolaeva, Relations politiques et circulations révolutionnaires entre l'Italie du Risorgimento et la Russie des autocrates et des réformateurs. Thèse en cours sous la direction de Gilles Pécout et Marie-Pierre Rey, Université Panthéon-Sorbonne.

25. Clément Thibaud, Républiques en armes. Les armées de Bolivar dans les guerres d'indépendance du Vénézuela et de la Bolivie, Rennes, PUR, 2006 ; Grégoire Bron, Révolution et nation entre le Portugal et l'Italie. Les relations politiques lusoitaliennes des Lumières à l'Internationale libérale de 1830, thèse de l'EPHE, 2013.

26. Simon Sarlin, Le légitimisme en armes. Histoire d'une mobilisation internationale contre l'unité italienne. Rome, École française de Rome, 2013.

27. Alexandre Dupont, Carlistes et légitimistes entre France et Espagne. Les relations entre contrerévolutionnaires français et espagnols dans la deuxième moitié du XIXe siècle. Thèse en cours sous la direction de Philippe Boutry, Université Panthéon-Sorbonne.

28. Jean Guénel, La dernière guerre du pape. Les zouaves pontificaux au secours du Saint-Siège, 1860-1870, Rennes, PUR, 1998, 195 p. 
publiques - question que Robert Frank élargit à celle des émotions, susceptibles d'influer sur les rapports entre les États, les peuples et les sociétés ${ }^{29}$ - montre l'importance de la politique étrangère dans la politisation des masses. En témoigne le rôle des opinions publiques franco-anglaises dans les crises qui agitent les années 1840 (crise d'Orient, affaire Pritchard), étudiées dans ce dossier par Renaud Meltz, ou plus tard, celui des opinions publiques en prise avec la politique officielle des Puissances face aux massacres arméniens de la fin du XIX ${ }^{\mathrm{e}}$ siècle $^{30}$.

Ces travaux introduisent dans l'histoire diplomatique des concepts et des savoirs venus de l'histoire culturelle ${ }^{31}$ et sociale ${ }^{32}$, mais aussi de la sociologie ${ }^{33}$ et des sciences politiques $^{34}$. Ils revisitent à nouveaux frais, au bénéfice du XIX ${ }^{\mathrm{e}}$ siècle, les questionnements les plus contemporains sur ce nouvel acteur des relations internationales. Aussi bien, les recherches sur l'opinion publique au $\mathrm{XIX}^{\mathrm{e}}$ siècle doivent beaucoup aux préoccupations et aux analyses du contemporain ; leur dette n'est pas moins importante à l'égard des historiens du Moyen Âge et de l'époque moderne ${ }^{35}$.

Or cette diplomatie des peuples repose sur la volonté de définir un bien universel, sur lequel fonder en raison, et en justice, chaque régime, et l'ensemble des nations, grâce à quoi la paix universelle sera permise. Ce désir d'appliquer la loi morale universelle au domaine des relations internationales n'est pas neuf, ni propre aux radicaux anglais ou aux républicains français. Il relaie, redouble ou croise des réflexions sur l'élaboration d'un corpus de règles coutumières et de conventions, connu sous le nom de droit des gens, jus gentium, inspiré du droit naturel qui s'est fait jour au XVII ${ }^{\mathrm{e}}$ siècle et dans la première moitié du siècle suivant, sous la plume de publicistes comme Puffendorf, Burlamaqui, Mably, Vattel. Sylvio De Francheschi montre leur importance, dans la double formation d'une

29. Robert Frank, «Émotions mondiales, internationales et transnationales, 1822-1932 », Revue Monde $(s)$, Histoire Espaces Relations, 2012/1, p. 47-70.

30. Vincent Duclert et Gilles Pécout, « La mobilisation intellectuelle face aux massacres d'Arméniens » dans Les Exclus en Europe 1830-1930, André Gueslin et Dominique Kalifa (dir.), Paris Editions de l'Atelier, 1999 , p. 323-344.

31. Dominique Kalifa, Philippe Régnier, Marie-Ève Thérenty et Alain Vaillant (dir.), La civilisation du journal, histoire culturelle et littéraire de la presse française au XIX ${ }^{e}$ siècle, Nouveau monde éditions, 2011 ; Françoise Parent-Lardeur, Les cabinets de lecture. La lecture publique à Paris sous la Restauration, Payot, 1982.

32. L'opinion : information, rumeur, propagande. Les Rendez-vous de l'histoire, Blois, 2007, Nantes, Pleins feux, 2007, et notamment l'introduction de Jean-Noël Jeanneney et les textes de Claude Gauvard, Christian Delporte, et Alain Corbin («L'opinion publique ou l'« état des esprits » du Premier empire à l'instauration du suffrage universel »). Christophe Charle, Le siècle de la presse, 1830-1939, Seuil, 2004.

33. Pierre Bourdieu, «L'opinion publique n'existe pas », Les Temps modernes, n 318, janvier 1973, p. 1295 1309 ; Loïc Blondiaux, La fabrique de l'opinion, une histoire sociale des sondages, Seuil, 1998 ; Matthieu Brugidou, L'opinion et ses publics, Une approche pragmatiste de l'opinion publique, Presses de la FNSP, 2008 (Voir la préface, très éclairante, de Nonna Mayer) ; Nicole d'Almeida (dir)., L'opinion publique, les Essentiels d'Hermès, CNRS éditions, 2009.

34. Dominique Reynié, Le triomphe de l'opinion publique. L'espace public français du XVI ${ }^{e}$ au XXe siècles, O. Jacob, 1998 ; Bertrand Badie, Le diplomate et l'intrus. L'entrée des sociétés dans l'arène internationale, Paris, Fayard, 2008.

35. L'opinion publique en Europe (1600-1800), PUPS, 2011, notamment l'introduction de Daniel Laroche, «L'opinion publique a-t-elle une histoire ? »; Keith Baker, Au tribunal de l'opinion. Essais sur l'imaginaire politique au XVIII siècle, Payot, 1993. Patrick Boucheron, Nicolas Offenstadt, L'espace public au Moyen Âge. Débats autour de Jürgen Habermas, PUF, 2011 ; Arlette Farge, Dire et mal dire. L'opinion publique au XVIII ${ }^{e}$ siècle, Le Seuil, 1992 ; Sandro Landi, Naissance de l'opinion publique dans l'Italie moderne, Sagesse du peuple et savoir de gouvernement de Machiavel aux Lumières, PUR, 2006. ; Mona Ozouf, L'homme régénéré. Essais sur la Révolution française, Gallimard, 1989 ( «Le concept d'opinion publique au XVIII ${ }^{\mathrm{e}}$ siècle »), p. 2153. 
tradition française d'une morale de raison, et d'un corpus d'ouvrages religieux, notamment de jésuites, sur les bonnes pratiques diplomatiques. Cette volonté de rendre conforme la conduite des rapports internationaux aux règles universelles de la raison et de la morale, traverse les temps et les partis. Elle entend répandre normes et pratiques européennes au-delà de son foyer : seule une histoire globale peut en rendre compte.

Comment écrire l'histoire diplomatique d'une Europe traversée par des mouvements transnationaux ${ }^{36}$, qui prétend disséminer ses valeurs, en se cantonnant au récit de l'entre-soi des États occidentaux ? Nous appelons de nos vœux cette « approche attentive aux formes plus subtiles et plus ténues de contacts entre les peuples ${ }^{37}$ », que ce soit par le biais des exilés, vecteurs des transferts culturels et politiques ${ }^{38}$, des volontaires internationaux et de toute la variété de forme des impérialismes. D'où l'importance accordée aux groupes de pression (missionnaires, cercles abolitionnistes, milieux pacifistes) et de tous ceux qui, à l'ombre des cabinets, ont agi et œuvré en faveur d'une vision morale des relations entre les États, au bénéfice des peuples et des individus. Si la diplomatie européenne occupe la première place, à l'image de sa domination de l'ordre international de l'époque, son prolongement par le biais des impérialismes et des logiques coloniales permet aussi de la confronter avec d'autres espaces (Empire Ottoman, le Golfe arabo-persique, Inde). À la suite des historiens des relations internationales nous souhaitons rompre avec une histoire européocentrée. L'approche d'une diplomatie interculturelle, définie par Christian Windler comme une « expérience de l'Autre », à propos des relations entre la France et les Échelles entre 1715 et $1840^{39}$, est naturellement féconde pour l'ensemble du XIX ${ }^{\mathrm{e}}$ siècle ${ }^{40}$. À côté du modèle étatique européen, il existe en effet des entités différentes, dotées d'autres statuts juridiques, qui ne disposent pas de tous les attributs de la souveraineté, mais participent pourtant du même système international comme le rappelle Fabrice Jesné ${ }^{41}$.

Ce dossier propose quelques contributions pour articuler diplomaties nationales et transnationales. L'article de Guillemette Crouzet laisse voir comment la condamnation de la traite produit une forme d'homogénéisation des pratiques et de socialisation des diplomaties. L'histoire diplomatique du XIX ${ }^{\mathrm{e}}$ siècle que nous avons en vue, se destine à l'étude des connexions et des interactions, à différentes échelles spatio-temporelles, afin de dépasser les « oppositions entre micro et macro et entre courte et longue durée pour mettre au point leurs imbrications ${ }^{42} »$ au bénéfice d'une histoire croisée. Pour ce faire, elle trouve

36. Christophe Charle «Les réseaux intellectuels européens au XIX $\mathrm{X}^{\mathrm{e}}$ siècle » dans Penser les frontières de l'Europe du XIX ${ }^{e}$ au XXI siècles, dir. Gilles Pécout, Editions Rue d'Ulm, PUF, 2004, p. 65-93.

37. A-C Ignace, Des Quarante-huitards français en Italie. op. cit., p. 21.

38. Delphine Diaz, Un asile pour tous les peuples ? Proscrits, exilés et réfugiés étrangers en France de 1813 à 1852. Thèse de l'Université Panthéon-Sorbonne, 2012.

39. Christian Windler, La diplomatie comme expérience de l'Autre. Consuls français au Maghreb (17001840), Genève, Droz, 2002.

40. A ce sujet, voir le débat autour de cet ouvrage dans Diplomaties, numéro spécial de Monde(s), Histoire, Espaces, Relations, 2014/5.

41. Fabrice Jesné, « Le consul, subalterne de la machine diplomatique ou intermédiaire par excellence ?» Débat autour de l'ouvrage de Christian Windler (La diplomatie comme expérience de l'Autre, op. cit.) dans Diplomaties, numéro spécial de Monde(s), Histoire, Espaces, Relations, 2014/5. Il cite notamment les entités créées par la colonisation, comme l'État libre du Congo, les territoires administrés par les compagnies à charte ou encore les dominions. On peut y ajouter les territoires sous domination ottomane, étudiés par Alexandre Massé, La « domination morale » : Les consuls de France dans l'Orient grec : Images, ingérences, colonisation (1815-1856). Thèse de l'Université de Toulouse 2, 2012.

42. Bénédicte Zimmerman, « Histoire comparée, histoire croisée », dans Historiographies, I. Concepts et débats, dir. Christian Delacroix, François Dosse, Patrick Garcia, Nicolas Offenstadt, Gallimard, coll. Folio Histoire, 2010, p. 174. 
de précieux renforts dans les outils méthodologiques et conceptuels de l'ensemble des sciences sociales - droit, sciences-politiques, anthropologie et sociologie ${ }^{43}-$, et de toute la discipline historique. L'histoire économique, culturelle (représentations) et sociale (microhistoire, prosopographie), permettent une analyse des réseaux ${ }^{44}$ et des jeux d'échelle, dans l'espace ${ }^{45}$ et dans le temps, par la combinaison de la micro-histoire et de la macro-histoire utilisées dans d'autres périodes.

Bref, le renouvellement historiographique repose avant tout sur un renouvellement épistémologique.

\section{Les pratiques diplomatiques au prisme de la morale : pour une histoire sociale et culturelle de la diplomatie}

Ce dossier reflète quelques facettes de ce renouvellement historiographique que nous appelons de nos vœux, en même temps que nous en recevons les premiers fruits. Les articles publiés dans ce numéro contribuent à divers titres à cette nouvelle épistémologie de l'histoire des pratiques diplomatiques ; l'angle de la morale y dispose, si l'on entend par morale des règles propres à définir une bonne diplomatie autant qu'un socle de valeurs communes pour la vie des États en bonne société.

Inutile, pour rehausser l'importance de ces nouvelles approches, de renier la centralité du fait politico-juridique, institutionnel, qui a longtemps régné en solitaire dans l'histoire diplomatique. Les relations inter-étatiques, étudiées sur la base des documents officiels, occupent légitimement un large pan de l'historiographie française actuelle ${ }^{46}$. La plupart des articles de ce dossier n'ignorent pas cette donnée élémentaire. Les auteurs n'hésitent pas à rouvrir, à nouveaux frais, des enquêtes sur des événements d'histoire politique, dont les récits les plus récents ont parfois près d'un siècle (voir les articles d'O. Varlan, E. Larroche, R. Meltz, ou G. Crouzet). Ce qui ne nous empêche pas d'ajouter une double exigence à ce constat élémentaire : la diplomatie n'est pas une affaire seulement politique ; par ailleurs, l'histoire politique ne suffit pas à rendre compte de toutes les dimensions politiques de la diplomatie. Ainsi, sans être contestée, la centralité du politique dans la diplomatie des États, au XIX ${ }^{\mathrm{e}}$ siècle, ne doit plus occulter la variété de ses domaines de compétence. Son accroissement et sa diversification spectaculaire, au $\mathrm{XX}^{\mathrm{e}}$ siècle ${ }^{47}$, en diplomatie culturelle, économique, technique ou sanitaire, ne peuvent pas se séparer des premiers jalons, posés au siècle précédent.

43. Peter Jackson, « Pierre Bourdieu, the « cultural Turn » and the Pratice of International History », Review of International Studies 34, 2008/1, p. 155-181. Parmi les auteurs les plus souvent exploités par les travaux d'histoire diplomatique, Fredrik Barth (Ethnic Groups and Boundaries : the Social Organization of Culture Difference, Bergen-Oslo: G. Allen and Unwin, 1970), Pierre Bourdieu (La distinction. Critique sociale du jugement, Éd. Minuit, 1979 ; «Les rites comme acte d'institution », Actes de la recherche en sciences sociales, $\mathrm{n}^{\circ} 43$, juin 1982, p. 58-63) ou Erving Goffman (Les rites d'interaction, Éd. Minuit, 1998 ; La mise en scène de la vie quotidienne? I-La présentation de soi. II- Les relations en public, Éd. Minuit, 1973).

44. Anne Claire Ignace analyse par exemple les réseaux de voisinage, de sociabilité et d'accueil, dans lesquels s'inséraient les 1000 à 1300 volontaires partis au secours de l'Italie démocratique durant le Quarantotto.

45. Outre la thèse de Christian Windler, d'autres travaux privilégient l'échelon local comme Mathieu Jestin, Le consulat de France à Salonique 1781-1912. Thèse en cours à l'Université Panthéon-Sorbonne sous la direction de Robert Frank et Anne Couderc ; id. «Les identités consulaires dans la Salonique ottomane, 1781-1912 », Monde(s), Histoire, Espaces, Relations, 2013/2, p. 189-209.

46. Jean-Claude Allain, Françoise Autran, Lucien Bély, Georges-Henri Soutou, Maurice Vaisse et al, Histoire de la diplomatie française, Perrin, 2005.

47. Laurence Badel et Stanislas Jeannesson (dir), Diplomaties en renouvellement, numéro spécial des cahiers Irice $2009 / 1\left(n^{\circ} 3\right)$. 
De plus, si les enjeux immuables de la diplomatie, puissance, sécurité, stratégies, alliances, continuent d'occuper la première place, ils sont relus ici dans une perspective culturelle, sous l'angle des « représentations et perceptions de soi comme de l'autre, individuelles et collectives, pour apprécier la prégnance des forces profondes et rendre compte in fine des comportements des décideurs ${ }^{48}$. » Dans ce dossier, Stanislas Jeannesson s'applique à lui-même cette exigence, dans son approche du solidarisme : Léon Bourgeois est à la fois prescripteur et usager d'une opinion publique actrice des relations internationales, ce qui modifie naturellement l'art diplomatique, que ce soit en termes d'information ou de négociation.

Comme l'écrit Lucien Bély, « une lecture anthropologique, sociologique ou culturelle de la réalité internationale et de l'action diplomatique s'immisce ainsi dans l'analyse historique $^{49}$. »Cette approche a été précocement empruntée par les historiens médiévistes et modernistes, dans le sillage des travaux de Lucien Bély et de Jean-Claude Waquet. Ceux-ci font une place à l'étude de la formation et de la culture des diplomates ${ }^{50}$, à la langue, l'écrit et le discours diplomatiques ${ }^{51}$, aux techniques de négociation ${ }^{52}$, aux circuits des savoirs et des pratiques ${ }^{53}$, aux réseaux de l'information et de la propagande. Ils sont plus particulièrement attentifs aux gestes et au cérémonial ${ }^{54}$, destinés non seulement à mettre en scène le prestige des États et à déterminer le rang des acteurs mais aussi à policer les rapports internationaux. Cette posture épistémologique est encore plus marquée par ceux d'entre eux qui rompent avec une histoire "statocentrée » et considèrent la

48. S. Jeannesson, « Diplomatie et politique étrangère de la France contemporaine », art. cit., p. 91.

49. L. Bély, « Les relations internationales », art. cit., p. 62.

50. M. Belissa, Fraternité universelle et intérêt national (1713-1795). Les cosmopolitiques du droit des gens, Kimé, 1998.

51. Stefano Andretta, Stephane Pequignot, Marie-Karine Schaub, Jean-Claude Waquet et Christian Windler (dir.), Paroles de négociateurs. L'entretien dans la pratique diplomatique de la fin du Moyen Âge à la fin du $X I X^{e}$ siècle, Rome, École française de Rome, 2010. Jean-Claude Waquet, « La lettre diplomatique. Vérité de la négociation et négociation de la vérité dans quatre écrits de Machiavel, du Tasse et de Panfilo Persico », dans Politique par correspondance. Les usages politiques de la lettre en Italie (XIV ${ }^{e}$-XVIII ${ }^{e}$ siècles), Dir. Jean Boutier, Sandro Landi, Olivier Rouchon, Rennes, PUR, 2009, p. 43-55.

52. L. Bély, Espions et ambassadeurs au temps de Louis XIV, op. cit. ; id., L'incident diplomatique aux $X V I^{e}$-XVIII $I^{e}$ siècles, Pedone, 2010 ; Jean-Claude Waquet, François de Callières. L'art de négocier en France sous Louis XIV, Éd. Rue d'Ulm, 2005 ; Claire Gantet, « L'institutionnalisation d'une négociation », Hypothèses 2000/1, p. 181-187 ; Marc Belissa, « Principes des négociations ou art de négocier ? », dans Publikationsportal Friedensverträge, (Veröffentlichung des Instituts für Europäische Geschichte, Mainz, Veröffentlichung der Projektgruppe Europäische Friedensverträge der Vormoderne), Mainz 2008-11-18, Abschnitt 1 - 16. http://www.iegmainz.de/publikationsportal/index.html.

53. Michel Espagne, Michael Werner (dir.), Transferts. Les relations interculturelles dans l'espace francoallemand : XVIII et XX ${ }^{e}$ siècles, Paris, Éd. Recherche sur les civilisations, 1988 ; Michel Espagne, Les transferts culturels franco-allemands, PUF, 1999 ; Marc Belissa et Eric Schnakenbourg, « Les circulations diplomatiques en Europe au XVIII ${ }^{\mathrm{e}}$ siècle : représentation, information, diffusion des modèles culturels », dans Les circulations internationales en Europe ; années 1680-années 1780, Actes du colloque de Bordeaux, 22 et octobre 2006, PUPS, 2011, p. 279-295.

54. Claire Gantet, La paix de Westphalie (1648). op. cit.; Barbara Stollberg-Rilinger, Les vieux habits de l'empereur : une histoire culturelle des institutions du Saint-Empire à l'époque moderne, Éd. de la Maison des Sciences de 1'homme, 2013 ; id., «Symbolische Kommunikation in der Vormoderne. Begriffe-ThesenForschungsperspektiven », Zeitschrift für Historische Forschung, vol. 31, 2004, p. 489-527 ; id., «Zeremoniell als politisches Verfahren. Rangordnung und Rangstreit als Strukturmerkmale des frühneuzeitlichen Reichstags », dans Neue Studien zur frühneuzeitlichen Reichsgeschichte, Dir. Johannes Kunisch, Berlin, Duncker \& Humblot, 1997, p. 91-132 ; Matthias Köhler, Strategie und Symbolik. Verhandeln auf dem Kongress von Nimwegen, Köln, Böhlau Verlag, 2011 ; Fabrice Brandli, Le nain et le géant. La République de Genève et la France au XVIII siècle, cultures politiques et diplomatie, Rennes, PUR, 2012. 
diplomatie avant tout comme un «ensemble de pratiques et d'expériences observables dans des zones de contact entre des entités politiques régies par des normes partiellement divergentes ${ }^{55} »$. Aussi l'histoire diplomatique s'ouvre-t-elle à l'histoire des interactions et des face-à-face, à l'analyse des pratiques culturelles qui accompagnent les différents niveaux de l'interaction, au poids des représentations culturelles de l'autre dans les prises de décision, aux conditions de production de normes communes, irréductibles à celles des États. Cette approche, enfin, permet de ne plus séparer l'histoire politique d'une histoire des idées et des idéologies longtemps sous-estimées par l'École française des relations internationales, influencée « par le primat de l'intérêt national et une conception strictement réaliste de la politique étrangère » selon les dires de Georges-Henri Soutou ${ }^{56}$, et actuellement en cours de réévaluation par l'historiographie ${ }^{57}$.

La notion de morale, comprise comme vertu désintéressée, nous place à l'articulation entre une histoire des idées, des normes et des pratiques : comment la diplomatie d'un pays peut-elle prétendre à la morale, à des valeurs universelles, puisqu'un État est intéressé, par définition, à ses intérêts particuliers de puissance ? Guillemette Crouzet montre que le Right to interfere de l'humanitarisme anglais, pris comme un tout, loin d'être purement désintéressé, a permis, en même temps que d'abolir la traite dans l'espace du golfe arabopersique, d'y établir un véritable lac britannique. L'intervention française en Espagne, décrite par Emmanuel Laroche, obéit autant à l'idéal de légitimité dynastique et de guerre morale, qu'à une volonté de puissance et d'influence, d'ambitions personnelles, bref de pratiques diplomatiques chahutées par les bouleversements de valeurs. De fait, l'expédition permet d'asseoir la légitimité de la Restauration. En revenant à la philosophie thomiste appliquée aux relations internationales, le Saint-Siège entend affirmer son rôle de médiation, qui le conforte en tant que puissance morale (cf. article de G. Ferragu). La confrontation de la Raison d'État et de la morale privée ou collective peut tout autant s'appliquer aux individus chargés de représenter l'État et censés aussi respecter une éthique professionnelle, qui résiste parfois difficilement aux appréciations personnelles de ce qu'il convient de faire pour bien servir son Prince et la paix comme le montre la trahison de Caulaincourt (cf. article d'O. Varlan). L'aventure sans précédent de l'Empire, dans un moment de bouleversement total des valeurs individuelles et politiques, a conduit Caulaincourt à suivre une morale personnelle, faute d'un code diplomatique adéquat. Pour autant, les grands bouleversements idéologiques du siècle n'ont pas pu ne pas influencer les codes moraux du diplomate.

Enfin, la recherche d'une morale universelle en relations internationales va de pair avec l'élaboration de normes et de justes conventions. La prise en compte progressive de l'opinion publique dans les relations internationales, notamment dans les monarchies constitutionnelles, permet la constitution d'un espace public européen certes étroit, mais qui favorise l'émergence d'une première opinion publique internationale (cf. article de R.

55. C. Windler, La diplomatie comme expérience de l'Autre, op. cit., p. 11.

56. G.H. Soutou, « Les relations internationales », op. cit, p. 280 ; Isabelle Davion et Stanislas Jeannesson, «Entretien avec Georges-Henri Soutou », dans Penser le système international, XIX ${ }^{e}$-XXI $I^{e}$ siècles, PUPS, 2013, p. 407.

57. G-H. Soutou, La guerre de cinquante ans. Les relations Est-Ouest, 1943-1990, Fayard, 2001 ; Christophe Dickès, Jacques Bainville et les Relations internationales, 1908-1936, Thèse de 1'Université Paris-Sorbonne, 2004 ; Marc Belissa, Fraternité Universelle et Intérêt National (1713-1795), op. cit. ; Virginie Martin, La diplomatie en Révolution. Structures, agents, pratiques et renseignements diplomatiques : l'exemple des diplomates français en Italie (1789-1796), Thèse de l'Université Panthéon-Sorbonne, 2011 ; Religion et diplomatie. Principes et expériences de l'action culturelle de la France au XXe siècle, Colloque organisé par Gilles Ferragu et Florian Michel le 13 décembre 2013 à l'université Panthéon-Sorbonne. 
Meltz). Elle explique aussi l'apparition d'un espace de droit dont le Golfe arabo-persique sous domination britannique a pu être un des laboratoires. C'est surtout sous l'effet de penseurs pacifistes et républicains internationalistes, de théoriciens comme Léon Bourgeois et de juristes, que se développe un droit émanant de la communauté internationale ${ }^{58}$ pour défendre les prérogatives des individus, contre la toute-puissance des États, désormais justifiables et passibles de sanctions ${ }^{59}$. La morale apparaît bien comme un des principaux pivots à partir desquels s'est opérée la transformation d'un droit des gens en un droit véritablement international, dont le $\mathrm{XIX}^{\mathrm{e}}$ siècle a été la charnière. Les deux conférences de la paix étudiées par S. Jeannesson ont permis d'inscrire dans le droit international, comme dans la pratique des relations internationales, des principes novateurs, comme les droits et obligations des États en temps de guerre, la codification et l'extension de la pratique de l'arbitrage comme moyen de règlement pacifique des conflits. Ainsi participent-elles, grâce à Léon Bourgeois, à l'avènement d'une " diplomatie du droit », seule capable d'assurer une paix durable. Les traités de paix de 1919 et la naissance de la Société des nations constituent à la fois l'aboutissement de ce processus et un nouveau départ, caractérisé par la promotion d'un nouveau modèle de sécurité, fondé sur les principes de justice, de droit et de responsabilité, ainsi que les règles de transparences, censées renouveler l'ordre international, dont la Grande Guerre avait démontré la faillite.

Aussi bien, ce dossier et, plus généralement, l'histoire que nous avons en vue, s'assigne de croiser les regards et les objets, pour embrasser tous les enjeux de la diplomatie : militaires et stratégiques, économiques et commerciaux, culturels et religieux, juridiques et humanitaires. Et de considérer l'objet « diplomatie » sous toutes ses formes : secrète et ouverte, privée et publique, officielle et officieuse. Ce numéro esquisse ainsi l'étude des voies informelles de négociation et d'action, relayées parfois par des acteurs non étatiques, et qui émergent aux côtés de la «diplomatie d'État » et de ses représentants officiels (cf. O. Varlan, R. Meltz et S. Jeannesson), thèmes explorés depuis quelques années par des travaux novateurs ${ }^{60}$.

Cette ambition inscrit l'histoire de la diplomatie du XIX ${ }^{\mathrm{e}}$ dans un temps long, en plongeant dans des débats et les usages nés à l'époque moderne (cf. l'article de $\mathrm{S}$. De Franceschi), nécessaires à l'élucidation d'un $\mathrm{XX}^{\mathrm{e}}$ siècle novateur en matière de principes et de pratiques. Ce choix permet de questionner le sens de la notion d'événement en histoire politique et d'interroger la pertinence des grandes ruptures - Révolution, Grande Guerre - en matière de valeurs et pratiques dans la conduite extérieure des États et de leurs représentants.

En somme, la notion de morale permet d'éprouver la pertinence et la possibilité de nos ambitions en faveur d'une histoire diplomatique renouvelée. L'histoire des idées ne suffit pas à rendre compte d'une diplomatie juste, dans ses principes et sa conduite ; nous avons essayé, ici, de la marier à une histoire des pratiques diplomatiques, qui ont

58. Dzovinar Kevonian, Réfugiés et diplomatie humanitaire : les acteurs européens et la scène procheorientale pendant l'entre-deux-guerres, Publications de la Sorbonne, 2004 ; id., «André Mandelstam and the internationalization of human rights (1869-1949) », dans Revisiting the origins of human rights : Genealogy of a European Idea, dir. Miia Halme-Toumisaari, Pamela Slotte, University of Helsinki, Erik Castren Institute of International Law and Human Rights, Cambridge University Press, 2014.

59. Jean-Michel Giueu et Dzovinar Kevonian (dir.), Juristes et relations internationales, numéro spécial de Relations internationales, 2012/1.

60. Par exemple, sur les acteurs économiques, Séverine Marin, L'apprentissage de la mondialisation : les milieux économiques allemands face à la réussite américaine, (1876-1914), Bruxelles, PIE Peter Lang, 2012 ; Laurence Badel, Un milieu libéral et européen : le grand commerce français, 1925-1948, Paris : ministère de l'Économie, des Finances et de l'Industrie, Comité pour l'histoire économique et financière de la France, 1999. 
leurs héritages spécifiques, évoluant à leur rythme propre, opposant une forme d'inertie aux bouleversements des idées, ou les bousculant par des novations relevant de l'histoire sociale - quoiqu' une diplomatie juste vise, selon le vœu de Guizot, à réunir buts et moyens dans la même moralité. L'évolution de l'ethos du diplomate, fondé sur des qualités privées mais universelles, héritées du système curial, n'évolue pas toujours au train des idées. La trahison ou la vénalité supposée de Caulaincourt, dont nous parle ici Olivier Varlan, nous oblige à l'appréhender sous l'ange des valeurs et des pratiques propres aux régimes, aux périodes, aux systèmes d'organisations internationales, sans que cette rationalisation ne puisse jamais épuiser le mystère d'une conduite individuelle, nourrie d'idéologie, de codes professionnels et de mobiles personnels. 